\title{
Cascaded VFO Control for Non-Standard N-Trailer Robots
}

\author{
Maciej Marcin Michałek • Marcin Kiełczewski • \\ Tomasz Jedwabny
}

Received: 16 January 2013 / Accepted: 20 May 2013 / Published online: 20 September 2013

(C) The Author(s) 2013. This article is published with open access at Springerlink.com

\begin{abstract}
Articulated mobile robots consisting of a tractor and passively off-hooked trailers belong to a class of highly nonlinear, nonholonomic, structurally unstable, differentially nonflat, and underactuated dynamic systems. Due to the mentioned properties of their kinematics, motion control problems related to $\mathrm{N}$-trailer robots ( $\mathrm{N}$-trailers) are non-trivial and challenging. Cascaded control strategy presented in this paper provides unified solution to the set-point and trajectory tracking control tasks for articulated robots equipped with arbitrary number of passively off-axle hitched trailers. Practically useful features of the proposed controller come from its high scalability and from application of the VectorField-Orientation (VFO) controller in the outer loop, which ensures fast errors convergence and simplicity of control implementation and tuning. Control input limitations of the robot are directly taken into account by utilization of a simple velocity scaling procedure which preserves an instantaneous motion curvature of a tractor. Description
\end{abstract}

This work was supported by the Polish scientific fund in years 2010-2012 as the research project No. N N514 087038.

M. M. Michałek $(\bowtie) \cdot$ M. Kiełczewski · T. Jedwabny Chair of Control and Systems Engineering,

Poznan University of Technology (PUT),

Piotrowo 3A, 60-965 Poznan, Poland

e-mail: maciej.michalek@put.poznan.pl and theoretical substantiation of the concept are followed by the results of experimental validation tests conducted with usage of a 3-trailer semiautonomous vehicle.

Keywords N-trailer robots • Off-axle hitching • Cascaded control $\cdot$ Set-point control .

Trajectory tracking

\section{Introduction}

The N-trailer robots (shortly: $\mathrm{N}$-trailers) consist of an active tractor-usually a differentially-driven cart-and arbitrary number of single-axle trailers interconnected by passive rotary joints. According to the type of interconnection between the vehicle segments (on-axle or off-axle) one distinguishes three kinds of tractor-trailer vehicles: Standard $\mathrm{N}$-Trailers (SNT) where all the hitching joints are situated at the midpoint of a preceding segment axle [10, 12], non-Standard N-Trailers (nSNT) where all the joints are mounted off the axle of a preceding segment [15], and General N-Trailers (GNT) with the mixed locations of particular hitching points-on and off the axles [1]. Highly nonlinear nature of N-trailer kinematics, the presence of nonholonomic constraints, structural instability of joint-angle subsystem, and nonminimum-phase properties resulting from off-axle interconnections [16] all make the motion control 
tasks of $\mathrm{N}$-trailers the non-trivial and challenging problems. Numerous, more or less specialized, solutions to different motion tasks have been proposed in the literature for 1-trailer or 2-trailer robots, cf. for instant [3-6, 8, 9, 11, 14, 17, 23, 27, 33-35]. On the other hand, much less results have been provided for $\mathrm{N}$-trailer vehicles with arbitrary number of segments (see for instant [22, 28, 29, 31, 32] where the control laws for SNT robots were proposed). The most sparse is the literature which tackles the motion control problems for nSNT and GNT vehicles [2, 13, 24]. It is a consequence of specific properties of their kinematics which are not differentially flat, and non-linearizable by feedback [1, 30]. Since availability of the results for nSNT and GNT vehicles is rather limited, further developments in this direction seem to be justified.

In the paper a highly scalable cascaded VFO controller for nSNT robots is presented and experimentally validated. Two classical motion tasks are considered-trajectory tracking (TT) and setpoint (SP) control. It is shown that the two tasks can be solved in a unified manner by using the cascaded control strategy together with the VectorField-Orientation (VFO) outer-loop controller. A structure of the proposed control system is a direct consequence of N-trailer kinematics, which can be formulated in the form of cascaded interconnection of unicycle subsystems with velocities resulting from propagation of tractor control inputs along a vehicle kinematic chain. Application of the cascaded control into nSNT robots is not a completely new idea-is was independently proposed in [7, 25] for the straight-line backward tracking task under a special assumption of a vehicle construction (where all the hitching distances and all the trailer lengths are equal), and in [21] for the backward path following task. In contrast, the approach proposed in the current paper presents a unified solution to the set-point and trajectory tracking control tasks for nSNT vehicles by utilization of the VFO controller [18] in an outer loop of a cascade. Solution admits backward and forward motion strategy for a vehicle. A set of admissible reference trajectories includes all the persistently exciting time-parametrized curves determined by a response of the unicycle model. The overall control performance is inherited from the outer-loop VFO controller, leading to fast errors convergence and simple (intuitive) synthesis of the resultant control system. Presentation of the concept is complemented by a set of experimental results acquired with the nS3T-type robotic vehicle on a laboratory testbed equipped with a vision feedback.

\section{Kinematics of $\mathrm{N}$-Trailer Vehicles and Control Problem Formulation}

\subsection{N-Trailer Kinematics in a Cascaded Form}

Kinematics of a non-standard N-trailer vehicle can be represented by the structure presented in Fig. 1. The vehicle consists of $N+1$ segments: an active differentially-driven tractor (with configuration and input components denoted by index zero) and $N$ passively off-axle hitched trailers numbered from 1 to $N$. Kinematic parameters of a vehicle are: trailer lengths $L_{i}>0$, hitching offsets ${ }^{1} L_{h i} \neq 0$ for all $i=1,2, \ldots, N$, tractor wheel radius $r>0$, and tractor wheel base $b>0$.

Configuration of the N-trailer can be uniquely determined by the vector

$\boldsymbol{q} \triangleq\left[\begin{array}{c}\boldsymbol{\beta} \\ \boldsymbol{q}_{N}\end{array}\right] \in \mathcal{Q} \subseteq \mathbb{R}^{N+3}$,

where

$\boldsymbol{\beta}=\left[\begin{array}{c}\beta_{1} \\ \vdots \\ \beta_{N}\end{array}\right] \in \mathcal{B} \subseteq \mathbb{R}^{N}, \quad \boldsymbol{q}_{N}=\left[\begin{array}{c}\theta_{N} \\ x_{N} \\ y_{N}\end{array}\right] \in \mathcal{Q}_{N} \subseteq \mathbb{R}^{3}$.

In the above definitions, $\boldsymbol{\beta}$ is the vector of relative joint angles, while $\boldsymbol{q}_{N}$ is the last-trailer configuration (posture) vector, which consists of the $N$-th trailer orientation angle $\theta_{N}$ and position coordinates $\left(x_{N}, y_{N}\right)$ of the guidance point $P$ selected in a midpoint of a trailer wheels-axle (cf. Fig. 1 for geometrical interpretation of particular variables). Location of the guidance point

\footnotetext{
${ }^{1}$ Note: a hitching offset is positive if it is located behind an axle of a preceding vehicle segment or negative if it is located in front of an axle.
} 


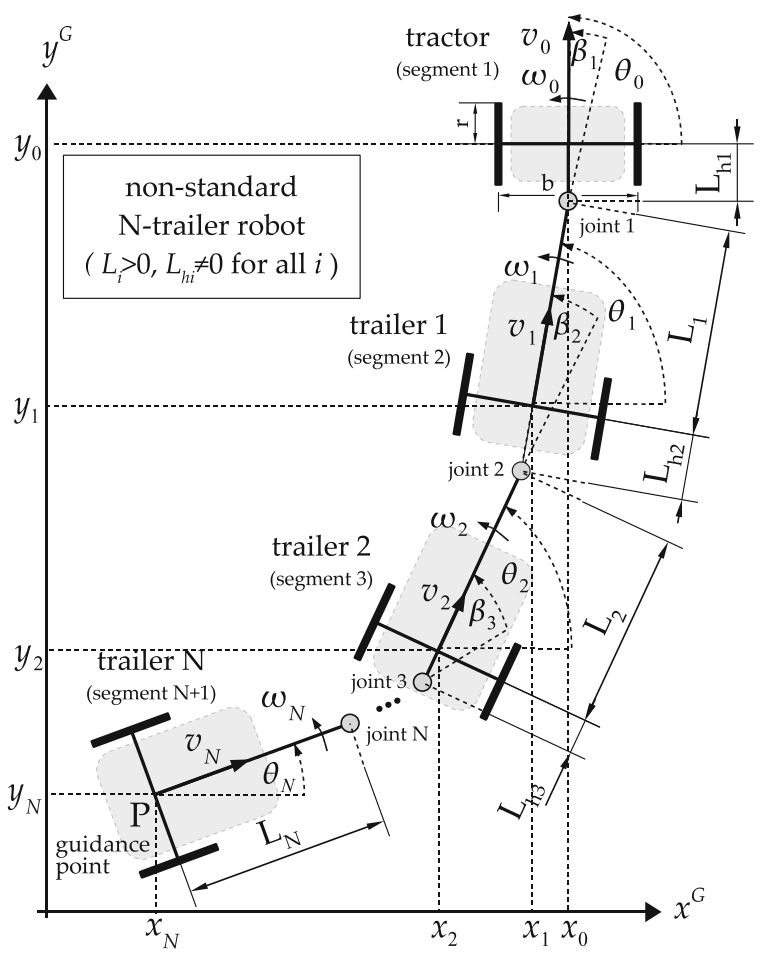

Fig. 1 Kinematic structure of a non-standard N-trailer robot with determination of configuration variables, control inputs, and kinematic parameters

is crucial from a viewpoint of a control task definition, which will be formulated in Section 2.2. The vehicle control input vector

$\boldsymbol{u}_{0} \triangleq\left[\omega_{0} v_{0}\right]^{\top} \in \mathcal{U} \subseteq \mathbb{R}^{2}$

consists of the angular and longitudinal velocities of a tractor. Control space $\mathcal{U}$ may be constrained to the subset of $\mathbb{R}^{2}$ due to physical (or more conservatively imposed by a user) limitations of the maximal admissible velocities of tractor wheels (actuators).

In order to obtain a kinematic model of the $\mathrm{N}$-trailer in a cascaded form let us treat each of the vehicle segments as a unicycle

$\dot{\boldsymbol{q}}_{i}=\boldsymbol{G}_{i}\left(\boldsymbol{q}_{i}\right) \boldsymbol{u}_{i}=\boldsymbol{g}_{1} \omega_{i}+\boldsymbol{g}_{2}\left(\boldsymbol{q}_{i}\right) v_{i}$,

where

$\boldsymbol{q}_{i}=\left[\begin{array}{c}\theta_{i} \\ x_{i} \\ y_{i}\end{array}\right], \quad \boldsymbol{G}_{i}\left(\boldsymbol{q}_{i}\right)=\left[\begin{array}{cc}1 & 0 \\ 0 & \cos \theta_{i} \\ 0 & \sin \theta_{i}\end{array}\right], \quad \boldsymbol{u}_{i}=\left[\begin{array}{c}\omega_{i} \\ v_{i}\end{array}\right]$, are the configuration vector, kinematic matrix, and virtual control input of the $i$-th segment, respectively. The orientation angle $\theta_{i}$ results from equation

$\beta_{i}=\theta_{i-1}-\theta_{i}$.

Kinematic relations between velocities of neighboring segments can be written in the matrix forms [15]

$\boldsymbol{u}_{i}=\boldsymbol{J}_{i}\left(\beta_{i}\right) \boldsymbol{u}_{i-1}, \quad \boldsymbol{u}_{i-1}=\boldsymbol{J}_{i}^{-1}\left(\beta_{i}\right) \boldsymbol{u}_{i}$,

with transformation matrices

$$
\begin{gathered}
\boldsymbol{J}_{i}\left(\beta_{i}\right)=\left[\begin{array}{cc}
-\frac{L_{h i}}{L_{i}} \cos \beta_{i} & \frac{1}{L_{i}} \sin \beta_{i} \\
L_{h i} \sin \beta_{i} & \cos \beta_{i}
\end{array}\right], \\
\boldsymbol{J}_{i}^{-1}\left(\beta_{i}\right)=\left[\begin{array}{cc}
-\frac{L_{i}}{L_{h i}} \cos \beta_{i} & \frac{1}{L_{h i}} \sin \beta_{i} \\
L_{i} \sin \beta_{i} & \cos \beta_{i}
\end{array}\right]
\end{gathered}
$$

well determined for nSNT vehicles since $L_{i}>0$ and $L_{h i} \neq 0$ for all $i=1, \ldots, N$. The set of Eqs. 4-8 represents cascaded kinematics of $\mathrm{N}$-trailer robot which is illustrated in the form of block scheme in Fig. 2. It is also possible to write the $\mathrm{N}$-trailer kinematics in an alternative closed form as a driftless nonholonomic system (cf. [15])

$\dot{\boldsymbol{q}}=\boldsymbol{S}(\boldsymbol{q}) \boldsymbol{u}_{0}$

with kinematic matrix $\boldsymbol{S}(\boldsymbol{q})$ obtained by combination of Eqs. 4-8 together with relation $\dot{\beta}_{i}=\omega_{i-1}-$ $\omega_{i}$ resulting from time-differentiation of Eq. 6 .

By using relations (7), one can express a velocity vector of any vehicle segment as a function of vehicle control input $\boldsymbol{u}_{0}$ or velocity vector $\boldsymbol{u}_{N}$ of the last trailer:

$$
\begin{aligned}
\boldsymbol{u}_{i} & =\prod_{j=i}^{1} \boldsymbol{J}_{j}\left(\beta_{j}\right) \boldsymbol{u}_{0} \\
& =\boldsymbol{J}_{i}\left(\beta_{i}\right) \boldsymbol{J}_{i-1}\left(\beta_{i-1}\right) \ldots \boldsymbol{J}_{1}\left(\beta_{1}\right) \boldsymbol{u}_{0}, \\
\boldsymbol{u}_{i-1} & =\prod_{j=i}^{N} \boldsymbol{J}_{j}^{-1}\left(\beta_{j}\right) \boldsymbol{u}_{N} \\
& =\boldsymbol{J}_{i}^{-1}\left(\beta_{i}\right) \boldsymbol{J}_{i+1}^{-1}\left(\beta_{i+1}\right) \ldots \boldsymbol{J}_{N}^{-1}\left(\beta_{N}\right) \boldsymbol{u}_{N} .
\end{aligned}
$$

Formulas (11)-(12), valid for any $i=1, \ldots, N$, represent, respectively, the backward and forward velocity propagation equations along a kinematic 
a u x l i a ry outputs ( joint angles)

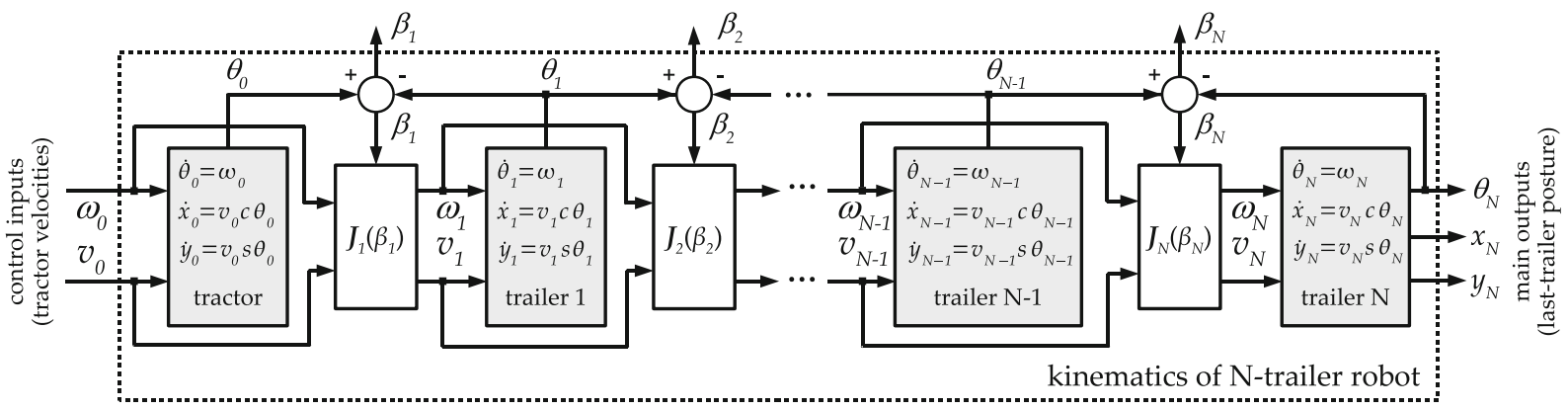

Fig. 2 Kinematics of N-trailer robots in the form of a multi-variable cascaded dynamic system (the model is valid for any type of N-trailer robots-SNT, nSNT, and GNT [15])

chain of nSNT vehicle. Velocity propagation formulas are essential for control law derivation presented in Section 3.

\subsection{Motion Tasks and Control Problem Statement}

Guidance point $P$ located on a last trailer makes the latter a guidance segment of the N-trailer robot. As a consequence, the motion tasks under consideration are to guide the last trailer configuration $\boldsymbol{q}_{N}(t)$ either to a constant reference posture (a set-point in configuration space $\mathcal{Q}_{N}$ )

$\boldsymbol{q}_{N r}=\left[\begin{array}{lll}\theta_{N r} & x_{N r} & y_{N r}\end{array}\right]^{\top} \in \mathcal{Q}_{N}$,

or along a desired time-varying reference trajectory

$\boldsymbol{q}_{N r}(t)=\left[\begin{array}{lll}\theta_{N r}(t) & x_{N r}(t) & y_{N r}(t)\end{array}\right]^{\top} \in \mathcal{Q}_{N}$.

From now on, we assume that reference trajectory (Eq. 14):

A1. is admissible by satisfying unicycle kinematics

$\dot{\boldsymbol{q}}_{N r}(t)=\boldsymbol{G}_{N}\left(\boldsymbol{q}_{N r}(t)\right) \boldsymbol{u}_{N r}(t)$

for some bounded and non-zero reference velocity $\boldsymbol{u}_{N r}(t)=\left[\omega_{N r}(t) v_{N r}(t)\right]^{\top}$,

A2. is at least twice differentiable $\left(\boldsymbol{q}_{N r}(t) \in C^{2}\right)$ and is persistently exciting in the sense that longitudinal reference velocity $v_{N r}(t) \neq 0$ for all $t \geq 0$.
In order to formulate the control problem in a mathematical manner let us introduce the posture error vector

$\boldsymbol{e}=\left[\begin{array}{l}e_{\theta} \\ e_{x} \\ e_{y}\end{array}\right]=\left[\begin{array}{c}e_{\theta} \\ \boldsymbol{e}^{*}\end{array}\right] \triangleq\left[\begin{array}{c}\mathcal{F}\left(\theta_{N r}-\theta_{N}\right) \\ x_{N r}-x_{N} \\ y_{N r}-y_{N}\end{array}\right] \in[-\pi, \pi) \times \mathbb{R}^{2}$,

where $\mathcal{F}: \mathbb{R} \mapsto[-\pi, \pi)$ maps the orientation error onto range $[-\pi, \pi)$. By $\boldsymbol{W} \triangleq \operatorname{diag}\{w, 1,1\} \in$ $\mathbb{R}^{3 \times 3}$ let us denote the weighting matrix with component $w \in(0,1]$ selected by a designer. The control problem is to design a feedback control law $\boldsymbol{u}_{0}=\boldsymbol{u}_{0}(\boldsymbol{e}, \cdot)$ which guarantees convergence of posture error (Eq. 16) in the sense that

$$
\|\boldsymbol{W} \boldsymbol{e}(t)\| \leq \delta \quad \forall t \geq T,
$$

for the prescribed precision $\delta \geq 0$, with $T \in(0, \infty)$ being the convergence time-horizon. Introduction of the weighting matrix $\boldsymbol{W}$ allows one to choose proportion between terminal values for particular error components of different units.

Formulation of the motion and control tasks in terms of a posture of only the guidance segment comes directly from practical reasons. In practice, a working implement is very often mounted on a last trailer. Loading/unloading tasks are also performed first for a last trailer in a chain. Backward maneuvers with trailers naturally makes a last trailer the guidance segment for a whole vehicle which can be treated as a virtual tractor in this case [20]. Therefore in practice, motion tasks with $\mathrm{N}$-trailers are usually prioritized where 
a posture of a last trailer is distinguished from a whole vehicle configuration, and stabilization of the rest configuration variables has a secondary meaning. Moreover, simultaneous control of all the configuration variables of $\mathrm{N}$-trailers (without any prioritization) can make a control process highly oscillatory with many tractor reversals, thus energetically expensive and unacceptable under practical conditions (see e.g. $[13,22,26]$ ).

\section{Cascaded Control Concept}

\subsection{Cascaded Control Law-General} Formulation

Cascaded structure of $\mathrm{N}$-trailer kinematics together with velocity propagation formulas (7) may suggest the following control design methodology.

According to Eq. 12 one can express an instantaneous desired tractor input, denoted by $\boldsymbol{u}_{0 c}$, as a function of an instantaneous desired velocity of the guidance segment $\boldsymbol{u}_{N c}$ :

$$
\boldsymbol{u}_{0 c}=\prod_{j=1}^{N} \boldsymbol{J}_{j}^{-1}\left(\beta_{j}\right) \boldsymbol{u}_{N c} .
$$

Let us make a thought experiment where, temporarily, the guidance segment is mechanically separated from the rest of the N-trailer. Furthermore, let us assume that some bounded control function $\boldsymbol{\Phi}(\boldsymbol{e}, \cdot) \in \mathbb{R}^{2}$ is given, which possesses the following properties:

P1. $\forall t \geq 0\|\boldsymbol{\Phi}(\boldsymbol{e}(t), \cdot)\| \leq M_{\phi}<\infty$,

$\mathrm{P} 2$. direct application of function $\boldsymbol{\Phi}(\boldsymbol{e}, \cdot)$ into the unicycle kinematics of the last-trailer ensures implication

$$
\begin{aligned}
\dot{\boldsymbol{q}}_{N} & =\boldsymbol{G}_{N}\left(\boldsymbol{q}_{N}\right) \boldsymbol{\Phi}(\boldsymbol{e}, \cdot) \\
& \Rightarrow \forall t \geq 0\|\boldsymbol{e}(t)\| \leq M_{e} \wedge\|\boldsymbol{e}(\tau)\| \stackrel{t \rightarrow \infty}{\longrightarrow} 0
\end{aligned}
$$

with upper bound $M_{e}<\infty$.

We do not determine any particular form of function $\boldsymbol{\Phi}(\boldsymbol{e}, \cdot)$ at the moment; it will be precisely defined based on the VFO approach in Section 3.2.
Since the guidance segment can be treated as a unicycle with virtual input $\boldsymbol{u}_{N}=\left[\begin{array}{ll}\omega_{N} & v_{N}\end{array}\right]^{\top}$, one may postulate to ensure that $\boldsymbol{u}_{N}=\boldsymbol{\Phi}(\boldsymbol{e}, \cdot)$ and, as a consequence, to force implication (19). Hence, let us define the desired velocity $\boldsymbol{u}_{N c} \triangleq \boldsymbol{\Phi}(\boldsymbol{e}, \cdot)$, and according to Eq. 18 the desired tractor input

$\boldsymbol{u}_{0 c}(\boldsymbol{e}, \boldsymbol{\beta}, \cdot)=\prod_{j=1}^{N} \boldsymbol{J}_{j}^{-1}\left(\beta_{j}\right) \boldsymbol{\Phi}(\boldsymbol{e}, \cdot)$.

Equation 20 represents a general definition of the cascaded control law for nSNT robots. Function $\boldsymbol{\Phi}(\boldsymbol{e}, \cdot)$ is an output of the last-trailer controller placed in an outer loop of a cascade (as illustrated in Fig. 3). Matrix transformation $\prod_{j=1}^{N} \boldsymbol{J}_{j}^{-1}\left(\beta_{j}\right)$ can be treated as the inner loop controller, which requires measuring of instantaneous values of joint angles $\beta_{i}, i=1, \ldots, N$, to propagate desired velocities from the last trailer toward the tractor segment. Let us summarize the cascaded control concept by the following proposition.

Proposition 1 Application to nSNT kinematics (Eq. 10) of a control input in the form

$\boldsymbol{u}_{0} \triangleq \boldsymbol{u}_{0 c}(\boldsymbol{e}, \boldsymbol{\beta}, \cdot)$

with desired control vector $\boldsymbol{u}_{0 c}(\boldsymbol{e}, \boldsymbol{\beta}, \cdot)$ determined by Eq. 20 and with outer-loop control function $\boldsymbol{\Phi}(\boldsymbol{e}, \cdot)$ having properties $P 1$ to $P 2$, ensures boundedness of input $\boldsymbol{u}_{0}$, and solves the control problem under consideration satisfying inequality (17) for $T=\infty$ if $\delta=0$ or $T<\infty$ if $\delta>0$.

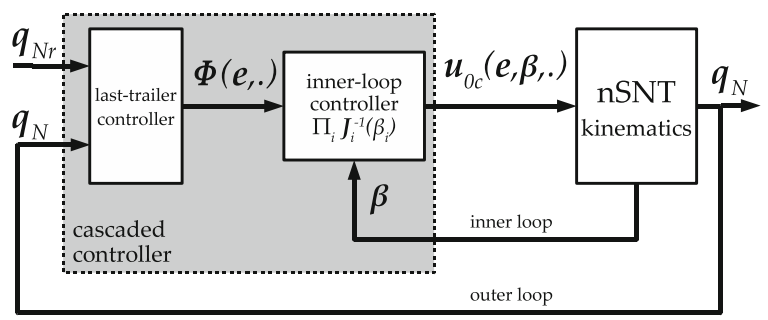

Fig. 3 General scheme of the proposed cascaded control system with the last-trailer (outer-loop) controller and the inner-loop controller responsible for velocity transformation along a vehicle kinematic chain 
Proof First, we will show boundedness of tractor control input $\boldsymbol{u}_{0}$. According to Eqs. 21, 20, and due to property $\mathrm{P} 1$ one can write what follows:

$$
\begin{aligned}
\left\|\boldsymbol{u}_{0}\right\| \stackrel{(21)}{=}\left\|\boldsymbol{u}_{0 c}(\boldsymbol{e}, \boldsymbol{\beta}, \cdot)\right\| \stackrel{(20)}{=}\left\|\prod_{j=1}^{N} \boldsymbol{J}_{j}^{-1}\left(\beta_{j}\right) \boldsymbol{\Phi}(\boldsymbol{e}, \cdot)\right\| \\
\leq \prod_{j=1}^{N}\left\|\boldsymbol{J}_{j}^{-1}\left(\beta_{j}\right)\right\|\|\boldsymbol{\Phi}(\boldsymbol{e}, \cdot)\| \leq \prod_{j=1}^{N} M_{j} M_{\phi}<\infty,
\end{aligned}
$$

where $M_{j}=\sqrt{\left(1+\frac{L_{j}^{2}}{L_{h j}^{2}}\right) \cos ^{2} \beta_{j}+\left(\frac{1}{L_{h j}^{2}}+L_{j}^{2}\right) \sin ^{2} \beta_{j}}$ is the Frobenius norm of matrix $\boldsymbol{J}_{j}^{-1}\left(\beta_{j}\right)$, which is bounded if only $L_{h j} \neq 0$ (it is satisfied for nSNT robots by definition).

Second, let us consider convergence of posture error $\boldsymbol{e}(t)$. By recalling propagation formula (11) one can show what follows:

$$
\begin{aligned}
& \boldsymbol{u}_{N} \stackrel{(11)}{=} \prod_{j=N}^{1} \boldsymbol{J}_{j}\left(\beta_{j}\right) \boldsymbol{u}_{0} \stackrel{(21)}{=} \prod_{j=N}^{1} \boldsymbol{J}_{j}\left(\beta_{j}\right) \boldsymbol{u}_{0 c}(\boldsymbol{e}, \boldsymbol{\beta}, \cdot) \\
& \stackrel{(20)}{=} \prod_{j=N}^{1} \boldsymbol{J}_{j}\left(\beta_{j}\right) \prod_{j=1}^{N} \boldsymbol{J}_{j}^{-1}\left(\beta_{j}\right) \boldsymbol{\Phi}(\boldsymbol{e}, \cdot) \equiv \boldsymbol{\Phi}(\boldsymbol{e}, \cdot) .
\end{aligned}
$$

The above result means that definition (Eq. 21) with the desired tractor input $\boldsymbol{u}_{0 c}(\boldsymbol{e}, \boldsymbol{\beta}, \cdot)$ determined by Eq. 20 guarantees, that at any time instant $t \geq 0$ the last-trailer velocity $\boldsymbol{u}_{N}$ is forced to be equal, as expected, to the instantaneous outer-loop control function $\boldsymbol{\Phi}(\boldsymbol{e}, \cdot)$. Hence, in the cascaded closed-loop system holds: $\dot{\boldsymbol{q}}_{N}=$ $\boldsymbol{G}_{N}\left(\boldsymbol{q}_{N}\right) \boldsymbol{\Phi}(\boldsymbol{e}, \cdot)$. By using property P2 with implication (Eq. 19) one concludes that the posture error $\boldsymbol{e}(t)$ is bounded and asymptotically converges toward zero as $t \rightarrow \infty$. Since all the weights in matrix $\boldsymbol{W}$ used in Eq. 17 are non-zero we have $\|\boldsymbol{W} \boldsymbol{e}\|=0 \Leftrightarrow\|\boldsymbol{e}\|=0$, thus one may conclude that $\|\boldsymbol{W} \boldsymbol{e}(t)\| \rightarrow 0$ as $t \rightarrow \infty$. The last statement corresponds to Eq. 17 with $\delta=0$ and $T=\infty$. As a consequence, for $\delta>0$ there exists a time instant $T<\infty$ such that $\|\boldsymbol{W} \boldsymbol{e}(t)\| \leq \delta$ for all $t \geq T$.

\subsection{Outer-loop VFO Controller}

To make cascaded control law (Eq. 20) complete, one needs to determine a form of the outerloop control function $\boldsymbol{\Phi}(\boldsymbol{e}, \cdot)$. We will define the outer-loop control function using the VFO control approach described in [18]. A general form of the VFO control function has a unified structure regardless the motion task is considered-SP or TT. It can be determined by the formula

$\boldsymbol{\Phi}(\boldsymbol{e}, \cdot)=\left[\begin{array}{c}\phi_{\omega}(\boldsymbol{e}, \cdot) \\ \phi_{v}(\boldsymbol{e}, \cdot)\end{array}\right] \triangleq\left[\begin{array}{c}h_{a}(\boldsymbol{e}, \cdot) \\ \boldsymbol{h}^{\top}(\boldsymbol{e}, \cdot) \boldsymbol{g}_{2}\left(\boldsymbol{q}_{N}\right)\end{array}\right]$,

where

$$
\begin{aligned}
\boldsymbol{h}(\boldsymbol{e}, \cdot) & =\left[\begin{array}{c}
h_{a}(\boldsymbol{e}, \cdot) \\
h_{x}(\boldsymbol{e}, \cdot) \\
h_{y}(\boldsymbol{e}, \cdot)
\end{array}\right]=\left[\begin{array}{l}
h_{a}(\boldsymbol{e}, \cdot) \\
\boldsymbol{h}^{*}(\boldsymbol{e}, \cdot)
\end{array}\right] \\
& \triangleq\left[\begin{array}{c}
k_{a} e_{a}\left(\boldsymbol{h}^{*}(\boldsymbol{e}, \cdot)\right)+\dot{\theta}_{a}\left(\boldsymbol{h}^{*}(\boldsymbol{e}, \cdot)\right) \\
k_{p} \boldsymbol{e}^{*}+\boldsymbol{v}^{*}(\cdot)
\end{array}\right]
\end{aligned}
$$

is the so-called convergence vector field defined with utilization of two design parameters $k_{a}>$ 0 and $k_{p}>0$. Particular components used in definition (25) take the forms: ${ }^{2}$

$$
\begin{aligned}
& e_{a}\left(\boldsymbol{h}^{*}(\boldsymbol{e}, \cdot)\right) \triangleq \theta_{a}\left(\boldsymbol{h}^{*}(\boldsymbol{e}, \cdot)\right)-\theta_{N} \\
& \theta_{a}\left(\boldsymbol{h}^{*}(\boldsymbol{e}, \cdot)\right) \triangleq\left\{\begin{array}{cc}
\operatorname{Atan} 2 \mathrm{c}\left(\sigma h_{y}, \sigma h_{x}\right) & \text { for }\left\|\boldsymbol{h}^{*}\right\|>0 \\
\theta_{a \lim } & \text { for }\left\|\boldsymbol{h}^{*}\right\|=0
\end{array}\right.
\end{aligned}
$$

$\dot{\theta}_{a}\left(\boldsymbol{h}^{*}(\boldsymbol{e}, \cdot)\right)=\left\{\begin{array}{cc}\frac{\dot{h}_{y} h_{x}-h_{y} \dot{h}_{x}}{h_{x}^{2}+h_{y}^{2}} & \text { for }\left\|\boldsymbol{h}^{*}\right\|>0 \\ 0 & \text { for }\left\|\boldsymbol{h}^{*}\right\|=0\end{array}\right.$,

where $A \tan 2 \mathrm{c}(\cdot, \cdot): \mathbb{R} \times \mathbb{R} \mapsto \mathbb{R}$ is a continuous version of the discontinuous function $\operatorname{Atan} 2(\cdot, \cdot)$ : $\mathbb{R} \times \mathbb{R} \mapsto[-\pi, \pi), \sigma \in\{-1,+1\}$ is the decision factor which determines a desired motion strategy for the guidance segment $(\sigma:=-1$ for backward motion or $\sigma:=+1$ for forward motion), and

$\theta_{a \lim } \triangleq \theta_{a}\left(\boldsymbol{h}^{*}(\boldsymbol{e}, \cdot) \rightarrow \mathbf{0}\right)$.

The term $v^{*}(\cdot)$ introduced in Eq. 25 has a meaning of a feedforward velocity; its form depends on the motion task considered and it is the only difference in the VFO control function (Eq. 23)

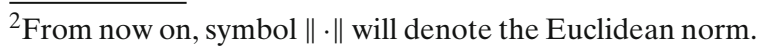


when it is used for the trajectory tracking or setpoint control task. The feedforward velocity is determined as follows:

$\boldsymbol{v}^{*}(\cdot)= \begin{cases}\boldsymbol{v}_{r}^{*}(t) & \text { for tracking control task, } \\ \boldsymbol{v}_{v}^{*}(\boldsymbol{e}) & \text { for set-point control task, }\end{cases}$

where

$v_{r}^{*}(t) \triangleq\left[\begin{array}{c}\dot{x}_{N r}(t) \\ \dot{y}_{N r}(t)\end{array}\right]$,

$\boldsymbol{v}_{v}^{*}(\boldsymbol{e}) \triangleq\left[\begin{array}{l}-\eta \sigma\left\|\boldsymbol{e}^{*}\right\| \cos \theta_{N r} \\ -\eta \sigma\left\|\boldsymbol{e}^{*}\right\| \sin \theta_{N r}\end{array}\right]$,

and $\eta \in\left(0, k_{p}\right)$ is an additional design parameter.

Remark 1 Switching conditions in Eqs. 27 and 28 have been introduced due to indeterminacy of $A \tan 2 c(0,0)$. Under conditions of measurement noises present in the outer feedback loop one may prefer to use a positive envelope $\epsilon$ in the switching conditions (instead of zero value) by selection:

$\epsilon \leq \delta \quad$ for SP control task

$0<\epsilon<\inf _{t}\left|v_{N r}(t)\right| \quad$ for TT control task.

In this case switching conditions in Eqs. 27 and 28 take the form of inequalities: $\left\|\boldsymbol{h}^{*}\right\|>\epsilon$ and $\left\|\boldsymbol{h}^{*}\right\| \leq \epsilon$, respectively, and $\theta_{a \lim }$ is the value of $\theta_{a}$ reached at the time instant of entering the envelope $\epsilon$ (cf. [18]).

Special structure of VFO control function (Eq. 23) originally results from geometrical interpretations of the unicycle kinematics and from decomposition of a unicycle control process into the azimuthal (related with component $\phi_{\omega}$ ) and radial (related with component $\phi_{v}$ ) control subprocesses [18]. In the geometrical interpretation, component $\phi_{\omega}$ can be called the orienting control function, while $\phi_{v}$ the pushing control function. In the case of a set-point control task the feedforward velocity $\boldsymbol{v}_{v}^{*}(\boldsymbol{e})$ defined by Eq. 32 is responsible for directing the unicycle motion in order to align its orientation with the reference one in a terminal control phase when the guidance segment approaches a reference position. Term $\boldsymbol{v}_{v}^{*}(\boldsymbol{e})$ is crucial in ensuring convergence of the orientation error toward zero. Intensity of the directing effect can be adjusted by a value of parameter $\eta$ (see Remark 2). More details on the VFO controllers can be found in [18]. The convergence analysis presented in [18] (cf. also [19]) revealed that the VFO control function (Eq. 23) has expected properties P1 to P2 for both TT and SP control tasks.

Having defined an outer-loop control function in the form of VFO controller (Eq. 23), the general cascaded control law (Eq. 21) will be hereafter called the cascaded VFO controller, and the resultant closed-loop system-the cascaded VFO control system (cf. Fig. 4).

Remark 2 Application of VFO control function (Eq. 23) requires selection of values for three design parameters $k_{a}, k_{p}$, and $\eta$. One can provide very simple heuristic tuning rules for the VFO controller (their effectiveness has been positively verified by numerous simulation and experimental tests [18]). The rules can be formulated as follows: $1^{\circ}$ select $k_{p}>0$ to get a compromise between the convergence rate for position error $\boldsymbol{e}^{*}(t)$, the resultant control cost, and sensitivity to feedback measurement noises (previous experience suggests $\left.k_{p} \in(0,5)\right), 2^{\circ}$ take $k_{a}=2 k_{p}$ because the orienting control process is crucial for the VFO control strategy, $3^{\circ}$ choose $\eta \in\left(0, k_{p}\right)$ according to an expected intensity of the directing effect by following the practical principle: the less the difference $\left(k_{p}-\eta\right)$, the greater intensity of directing [18]. The two-valued decision factor $\sigma \in$ $\{-1,+1\}$ used in Eqs. 27 and 32 determines the desired motion strategy for the guidance segment. In the case of trajectory tracking task one should select value of decision factor

$\sigma:=\operatorname{sgn}\left(v_{N r}(t)\right)$,

where $v_{N r}(t)$ is the reference longitudinal velocity along reference trajectory $\boldsymbol{q}_{N r}(t)$ (cf. Eq. 15). According to assumption A2 we have $v_{N r}(t) \neq 0$ for all $t \geq 0$, thus the right-hand side of Eq. 35 is constant and non-zero for all $t \geq 0$. For the set-point control task decision factor $\sigma$ can be freely chosen by a designer for almost all initial conditions $\boldsymbol{e}(0)$ (cf. [15]).

Remark 3 The hints on the decision factor selection formulated in Remark 2 correspond to the 
case when the original VFO controller can be directly applied into the unicycle kinematics. In the case where the VFO controller is utilized in the cascaded control law proposed in Section 3.1, there may exist limitations in selection of $\sigma$ value for some practical motion conditions. Limitations allow preventing the effect of vehicle folding in particular joints, which has to be avoided in most practical cases (due to mechanical constraints imposed on admissible joint angles). The issue of vehicle folding has been treated in details in [15]. The general rule of $\sigma$ selection (to avoid the folding effect) is to satisfy equality

$\sigma=-\operatorname{sgn}\left(L_{h i}\right)$.

The above requirement implies that the control tasks can be accomplished without the vehicle folding only in the backward strategy in case of positive hitching offsets, or only in the forward strategy in case of negative offsets. Furthermore, since we have in our disposal only a single decision factor, all the vehicle hitching offsets must be of the same sign. The latter fact imposes the assumption about homogeneous hitching of all the trailers. $^{3}$

\subsection{Addressing Control Input Limitations}

Control input limitations of the N-trailer result from a maximal admissible angular velocity imposed on the tractor wheels. In this case, control space $\mathcal{U}$ introduced in Eq. 3 has a diamond-like shape indicating that the instantaneous admissible values of angular and longitudinal tractor velocities are interrelated [15]. Thus, one needs to consider input limitations in the wheel-velocity space which has a square shape. Let us denote by $\omega_{w \max }>0$ the maximal admissible angular velocity of the tractor wheel which cannot be exceeded during vehicle motion. ${ }^{4}$ Transformation of the nominal control vector $\boldsymbol{u}_{0 c}$ computed by

\footnotetext{
${ }^{3}$ Note: in case where the vehicle folding is admissiblethere are no constraints on vehicle joint angles-the homogeneous hitching assumption and restriction (36) can be completely annulled.

${ }^{4}$ Value of $\omega_{w \max }$ can result from physical limitations of vehicle actuators or may be more conservatively selected due to the motion safety or energy-saving reasons.
}

Eq. 20 can be transformed to the wheel velocity space through the linear map

$\boldsymbol{\omega}_{0 c}(t)=\left[\begin{array}{l}\omega_{0 R c}(t) \\ \omega_{0 L c}(t)\end{array}\right]=\boldsymbol{T}^{-1} \boldsymbol{u}_{0 c}(t)$,

where

$\boldsymbol{T}=\left[\begin{array}{cc}r / b & -r / b \\ r / 2 & r / 2\end{array}\right]$

is the constant transformation matrix (parameters $r$ and $b$ are the tractor wheel radius and the tractor wheel base, respectively). Vector $\omega_{0 c}(t)$ includes current desired velocities $\omega_{0 R c}(t)$ and $\omega_{0 L c}(t)$ for the right and left wheel, respectively. In general, values of $\omega_{0 R c}(t)$ and $\omega_{0 L c}(t)$ may violate limitation $\omega_{w \max }$. Thus, to guarantee satisfaction of the limit, one introduces scaling function $k_{s}: \mathbb{R}^{2} \mapsto(0,1]$ in the form

$k_{s}\left(\omega_{0 c}(t)\right) \triangleq \frac{1}{s\left(\omega_{0 c}(t)\right)}$,

where

$s\left(\omega_{0 c}(t)\right) \triangleq \max \left\{1 ; \frac{\left|\omega_{0 R c}(t)\right|}{\omega_{w \max }} ; \frac{\left|\omega_{0 L c}(t)\right|}{\omega_{w \max }}\right\}$.

The nominal control vector $\boldsymbol{u}_{0 c}(t)$ can be modified on-line (for every time instant $t \geq 0$ ) leading to the scaled control vector

$\boldsymbol{u}_{0 s}(t) \triangleq k_{s}\left(\boldsymbol{\omega}_{0 c}(t)\right) \boldsymbol{u}_{0 c}(t)$.

Equations 38-40 represent the velocity scaling procedure. Note that control vector $\boldsymbol{u}_{0 s}(t)$ has a decreased norm (in comparison to $\left\|\boldsymbol{u}_{0 c}(t)\right\|$ ), but it preserves a direction and a sense of the nominal vector $\boldsymbol{u}_{0 c}(t)$ at any time instant. As a consequence, the instantaneous desired motion curvature $\kappa_{0 c}(t)=\omega_{0 c}(t) / v_{0 c}(t)$ for the tractor is preserved: $\kappa_{0 s}(t)=\omega_{0 s}(t) / v_{0 s}(t)=\kappa_{0 c}(t)$. Furthermore, desired velocities $\left[\omega_{0 R s} \omega_{0 L s}\right]^{\top}=\boldsymbol{T}^{-1} \boldsymbol{u}_{0 s}$ do satisfy limitation determined by the upper bound $\omega_{w \max }$. The last two statements can be summarized by the corollary

$\forall t \geq 0 \quad \frac{\omega_{0 c}(t)}{v_{0 c}(t)}=\frac{\omega_{0 s}(t)}{v_{0 s}(t)} \wedge\left|\omega_{0 R, L s}(t)\right| \leq \omega_{w \max }$,

which is valid after application of the scaling procedure (Eqs. 38-40). In the tractor-input space 


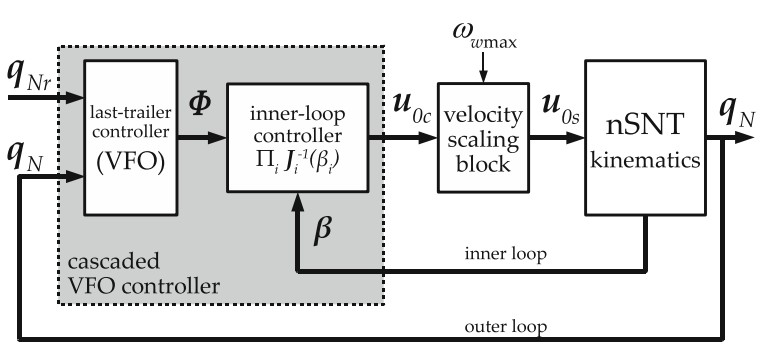

Fig. 4 Block scheme of the proposed cascaded VFO control system with the velocity scaling block serially placed between the cascaded controller and nSNT kinematics

it guarantees that $\forall t \geq 0\left|\omega_{0}(t)\right| \leq \omega_{0 \max },\left|v_{0}(t)\right| \leq$ $v_{0 \max }$, where

$\omega_{0 \max }=\frac{2 r}{b} \omega_{w \max }, \quad v_{0 \max }=r \omega_{w \max }$.

Utilization of the velocity scaling procedure is explained in Fig. 4, where the velocity scaling block has been placed in the main route between the cascaded VFO controller and the nSNT kinematics. Application of the scaling procedure has a serious (and beneficial) impact to a resultant control performance.

\section{Report of Experimental Validation}

\subsection{Testbed Description}

Experimental RMP-SW testbed consists of two main components. The first one is the 3-trailer RMP robot (presented in Fig. 5) equipped with two brushless DC motors (8-pole-pair, $50 \mathrm{~W}$, Maxon EC 45-flat with gearboxes of ratio $47: 1$ ). Each motor is independently controlled by the PI speed regulator with feedback from hall-sensors. Values of tractor kinematic parameters are: wheel radius $r=0.0293 \mathrm{~m}$, and wheel base $b=0.15 \mathrm{~m}$. All three trailers have the same lengths $L_{i}=0.229 \mathrm{~m}, i=1,2,3$. Location of the vehicle joints are mechanically adjustable in range $[-0.008 ; 0.056] \mathrm{m}$ with resolution of $0.008 \mathrm{~m}$ (hitching offsets can be made positive, zero or negative). Joint angles are measured by 14-bit absolute encoders (Hengstler AD36). RMP robot is equipped with the on-board DSP floating-point processor (TMS320F28335), which makes the robot computationally self-sufficient (the cascaded control law and reference signals are computed in real time on the board). Two independent incremental encoders (MHK40-8-2000-5-N) with resolution of $2000 \mathrm{imp} / \mathrm{rev}$ are mounted on the wheel semi-axles in the last trailer providing measurements for dead reckoning.

The second component of RMP-SW testbed is an external vision system, which consists of a digital camera (uEye UI-1240SE-C, resolution $1280 \times 1024$, sampling $25 \mathrm{~Hz}$ ) connected to an external PC computer. Vision system plays a role of an exteroceptive localization sensor, which on-line estimates a posture of the guidance segment based upon a current view of a LED marker located on the last trailer (cf. Fig. 5). Resolution of the vision localization is $0.4 \mathrm{~mm}$ for position coordinates and $0.3 \mathrm{deg}$ for an orientation angle. Exemplary (focused) view from the external camera together with a result of the last-trailer posture estimation are presented in Fig. 6. Wireless communication (modules CC2500 TI) between the robot and the external PC computer allows for closure of a vision feedback, and for exchange of signal samples (for visualization purposes) and user-selected system parameters.

\subsection{Implementation Details}

A block scheme of the cascaded control system implemented on the RMP-SW testbed is presented in Fig. 7. On the scheme one can distinguish two main computational subsystems synchronized by two different sampling periods: $T_{p}=0.01 \mathrm{~s}$ for all the computations performed by the on-board processor, and $T_{d}=4 T_{p}=0.04 \mathrm{~s}$ for computations related with the external vision system..$^{5}$ Two independent subsystems which are responsible for real-time estimation of the lasttrailer posture have been implemented in the system. The first subsystem performs exteroceptive estimation providing the absolute posture estimate $\hat{\boldsymbol{q}}_{N e}$ computed based on the LED marker localization by the vision system. The second subsystem performs proprioceptive estimation by incremental

\footnotetext{
$\overline{{ }^{5} \text { In RMP-SW }}$ system the average time of vision signal processing and exteroceptive posture estimation is about $0.008 \mathrm{~s}$.
} 


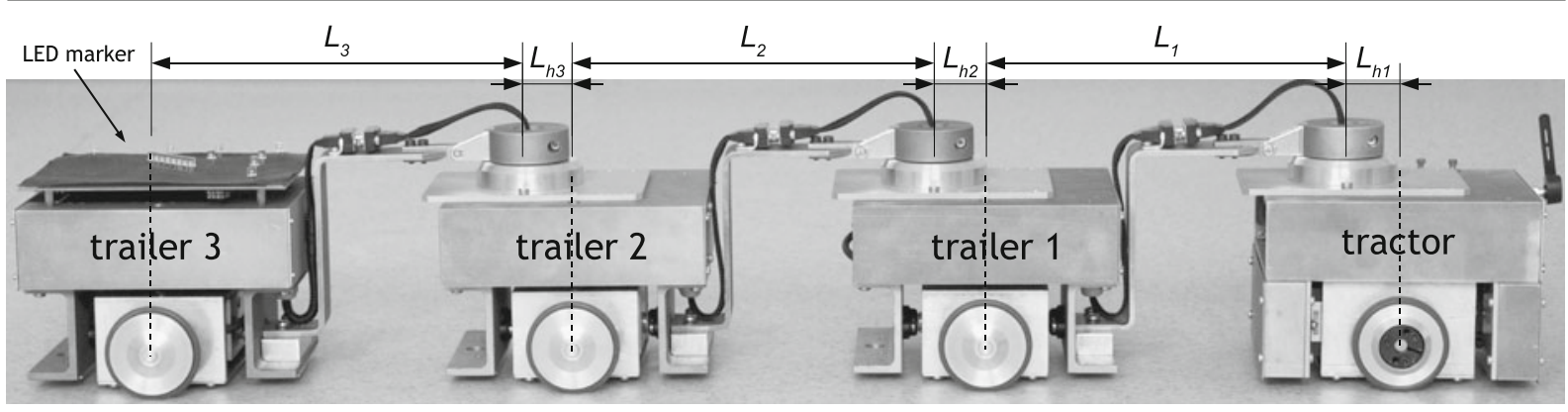

Fig. 5 RMP articulated mobile robot used during experimental validation tests (nS3T version is shown with hitching offsets $\left.L_{h i}>0, i=1,2,3\right)$

computations of posture $\hat{\boldsymbol{q}}_{N p}$ based on the variables or measurements accessible on the robot board. Proprioceptive estimation is a result of numerical integration of the unicycle kinematics (Eq. 4) for $i=N$, which can be approximated by the Euler method with a constant sampling time $T_{p}$ as follows:

$\hat{\boldsymbol{q}}_{N p}(n) \approx \hat{\boldsymbol{q}}_{N}(n-1)+T_{p} \cdot \boldsymbol{G}_{N}\left(\hat{\boldsymbol{q}}_{N}(n-1)\right) \tilde{\boldsymbol{u}}_{N}(n-1)$,

where $\hat{\boldsymbol{q}}_{N}$ denotes the estimate determined by Eq. 42 , and input $\tilde{\boldsymbol{u}}_{N}(n-1)$ can be selected as a one of two possible options ( $\mathrm{PbE}$ or $\mathrm{MbE})$ :

$\tilde{\boldsymbol{u}}_{N}(n-1):=\left\{\begin{array}{ll}\prod_{i=N}^{1} \boldsymbol{J}_{i}\left(\beta_{i}\right) \boldsymbol{u}_{0 s}(n-1) & \text { for } \mathrm{PbE} \\ \hat{\boldsymbol{u}}_{N}(n-1) & \text { for } \mathrm{MbE}\end{array}\right.$.

In the above formulation $\mathrm{PbE}$ means the Prediction-based Estimator (input $\tilde{\boldsymbol{u}}_{N}$ is predicted

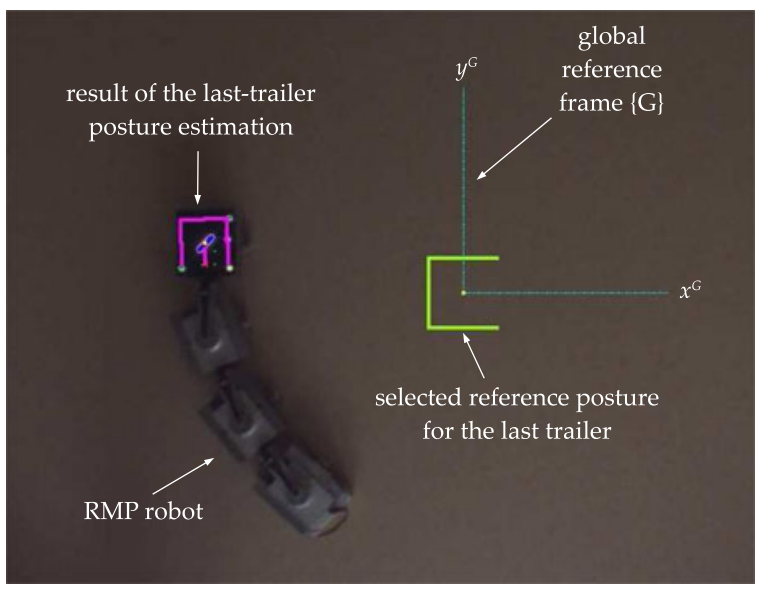

Fig. 6 Exemplary view from the external digital camera used on the experimental testbed together with a result of the last-trailer posture estimation according to the tractor control input $\boldsymbol{u}_{0 s}$ computed by the cascaded control law, and propagated using formula (11)), while MbE means the Measurement-based Estimator (input $\tilde{\boldsymbol{u}}_{N}$ is estimated according to measurements of velocities of the last-trailer wheels). Selection between $\mathrm{PbE}$ and $\mathrm{MbE}$ depends on a designer.

The results from the two subsystems $\left(\hat{\boldsymbol{q}}_{N e}\right.$ and $\hat{\boldsymbol{q}}_{N p}$, respectively) are fused in order to obtain the resultant posture estimation $\hat{\boldsymbol{q}}_{N}$ with improved quality. Estimate $\hat{\boldsymbol{q}}_{N}$ is ready for use as an outerloop feedback signal. The fusion mechanism can be explained as follows.

Let $t_{p} \triangleq n T_{p}$ and $t_{d} \triangleq m T_{d}, n, m \in \mathbb{N}$, denote the two time variables related with two sampling intervals $T_{p}$ and $T_{d}=4 T_{p}$, respectively. The resultant posture estimate $\hat{\boldsymbol{q}}_{N}$ at time instant $t_{p}$ is obtained according to equation

$\hat{\boldsymbol{q}}_{N}\left(t_{p}\right):= \begin{cases}\hat{\boldsymbol{q}}_{N p}\left(t_{p}\right) & \text { if } t_{p} \neq t_{d} \\ w_{1} \cdot \hat{\boldsymbol{q}}_{N p}\left(t_{p}\right)+w_{2} \cdot \hat{\boldsymbol{q}}_{N e}\left(t_{d}\right) & \text { if } t_{p}=t_{d}\end{cases}$

with non-negative weights $w_{1}$ and $w_{2}$ satisfying relation $w_{1}+w_{2}=1$. Equation 42 indicates that the proprioceptive estimate is combined with the vision estimate with period $T_{d}$. Within time intervals $\left(n T_{p} ;(n+3) T_{p}\right]$ posture estimate $\hat{\boldsymbol{q}}_{N}$ is equivalent to the proprioceptive estimate. Values of the weights $w_{1}, w_{2}$ are selectable by a designer as a compromise between filtration effectiveness of the high frequency measurement noise present in estimate $\hat{\boldsymbol{q}}_{N e}$, and a level of a drift characteristic for the proprioceptive estimate $\hat{\boldsymbol{q}}_{N p}$. 
Fig. 7 Cascaded control system implemented on the RMP-SW testbed with vision feedback; the red arrows indicate components of the outer feedback loop, and the blue arrow denotes the inner feedback loop

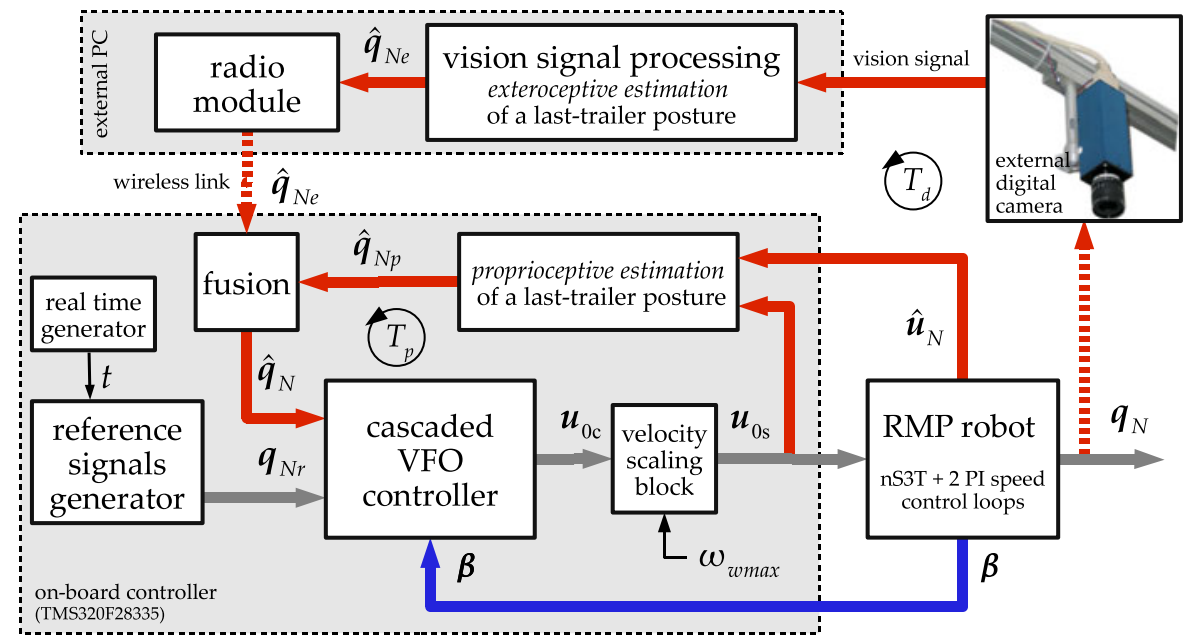

\subsection{Results and Comments for Set-point Control Task}

Two sets of set-point control experiments, E1 and E2, have been conducted for nS3T vehicle with negative and positive hitching offsets, respectively. In both experiments three scenarios of parking maneuvers (perpendicular-, parallel-, and U-turn-parking) has been presented for different initial conditions of the robot. In all cases the outer feedback loop was computing according to fusion mechanism (Eq. 42) with utilization of the $\mathrm{PbE}$.

Experiment E1 In experiment E1 negative hitching offsets $L_{h 1}=L_{h 2}=L_{h 3}=-0.008 \mathrm{~m}$ were adjusted in the robot. During first control trials highly oscillatory movement of the tractor was observed as a consequence of very small hitching offset values used in inverse matrices (Eq. 9). Since the hitching offsets affect mainly angular velocities in transformation formula (12) (cf. the first row of matrix in Eq. 9), high oscillations and permanent saturations of the angular control component of input $\boldsymbol{u}_{0}$ were observed after application of the scaling procedure (Eq. 40). As a consequence, the overall vehicle motion was highly non-smooth and simultaneously sluggish due to very small values of the tractor longitudinal velocity possible under these conditions. In order to attenuate mentioned oscillations, the overestimated values of hitching offsets $L_{h 1}=L_{h 2}=L_{h 3}=-0.032 \mathrm{~m}$ were used in computations of the inner-loop controller and in the $\mathrm{PbE}$. The following parameters were chosen for the remaining control blocks: $k_{a}=2, \quad k_{p}=1, \quad \eta=0.6, \quad \sigma=-\operatorname{sgn}\left(L_{h i}\right)=+1$ (forward parking maneuvers), and $\omega_{w \max }=$ $6 \mathrm{rad} / \mathrm{s}$. The weights in Eq. 42 were set to $w_{1}=0.98, w_{2}=0.02$. Since in practice one cannot expect precise convergence of the posture error to zero, the SP control task was terminated according to the switching strategy:

$\boldsymbol{\Phi}(\boldsymbol{e}):=\left\{\begin{array}{cl}(23) & \text { for }\|\boldsymbol{W} \boldsymbol{e}(t)\|>\delta, \\ \mathbf{0} & \text { for }\|\boldsymbol{W} \boldsymbol{e}(t)\| \leq \delta,\end{array}\right.$

with $\delta=0.02$ and $\boldsymbol{W}=\operatorname{diag}\{\sqrt{0.001}, 1,1\}$ (note that Eq. 43 still ensures satisfaction of inequality (17)). The results of experiment E1 are presented in Figs. 8 and 9. Three scenarios (A, B, and C) of subsequent parking maneuvers have been shown, where the reference postures (values in [rad, $\mathrm{m}, \mathrm{m}]$ )

$\boldsymbol{q}_{3 r}^{A}=\left[\begin{array}{c}-\pi \\ -0.4 \\ 0.0\end{array}\right], \quad \boldsymbol{q}_{3 r}^{B}=\left[\begin{array}{c}0.0 \\ 0.9 \\ -0.7\end{array}\right], \quad \boldsymbol{q}_{3 r}^{C}=\left[\begin{array}{c}\frac{\pi}{2} \\ 1 \\ 1\end{array}\right]$

have been denoted in Fig. 8 in the form of green dock-marks. Initial robot configurations $\boldsymbol{q}(0)$ have been highlighted in magenta.

Analyzing time plots in Fig. 9 one can find nonoscillatory convergence of the guidance segment toward the reference postures. Control performance for the last trailer directly results from properties of the VFO control law used in the 

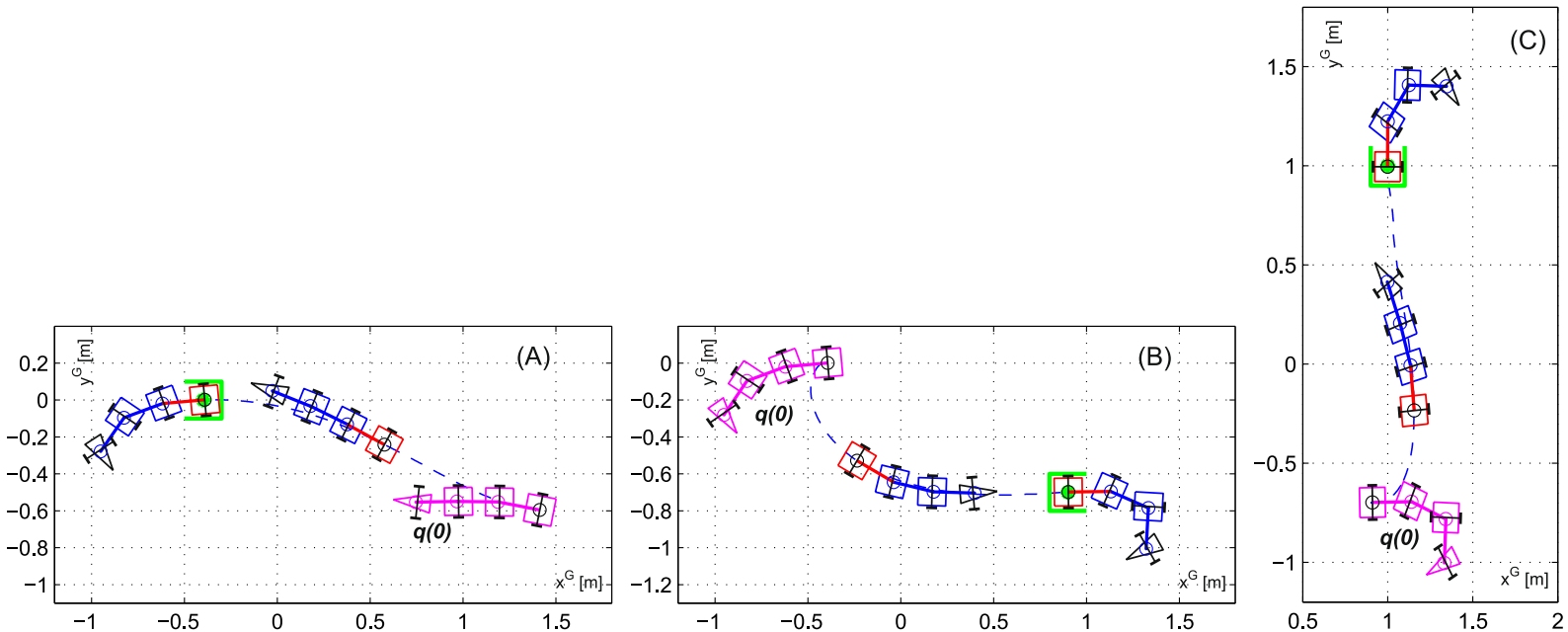

Fig. 8 E1: experimental results of three forward parking maneuvers $(\mathbf{A}, \mathbf{B}$, and $\mathbf{C})$ performed in a task space with $\mathrm{nS} 3 \mathrm{~T}$ robot in the case of negative hitching offsets
$\left(L_{h 1,2,3}<0\right)$; initial robot configurations $\boldsymbol{q}(0)$ are highlighted in magenta, and reference postures $\boldsymbol{q}_{3 r}$ are represented by green dock-marks

in the first robot joint. This sensitivity grow can be explained by the form of inverse matrix in Eq. 9. For $L_{h i} \approx 0$ the transformation matrix $\boldsymbol{J}_{i}\left(\beta_{i}\right)$ becomes close to singular one, thus any noises present in the outer loop are substantially gained by the inner loop. Despite oscillatory behavior of the joint angles, any folding effect has not occurred during parking maneuvers. Further-
(A)
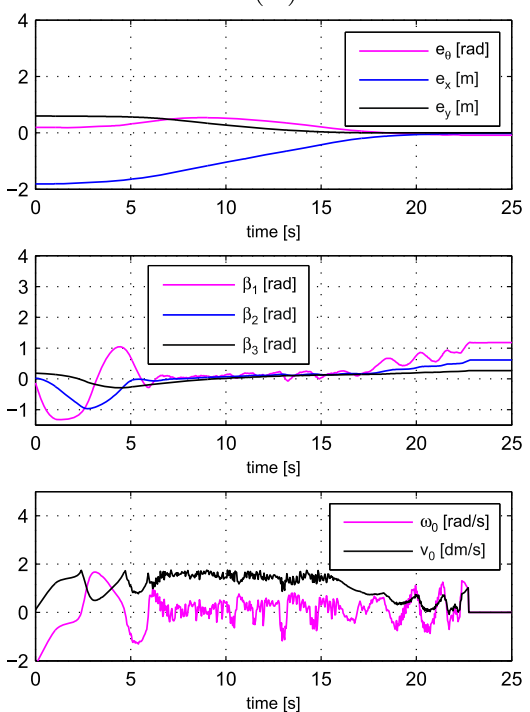

(B)
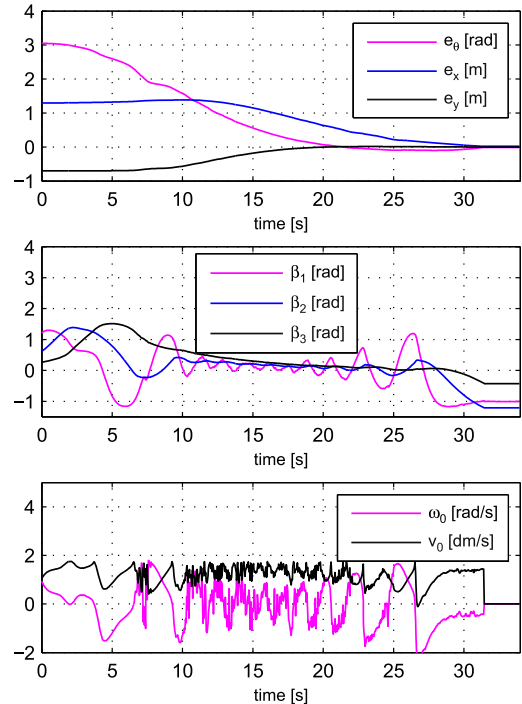

(C)
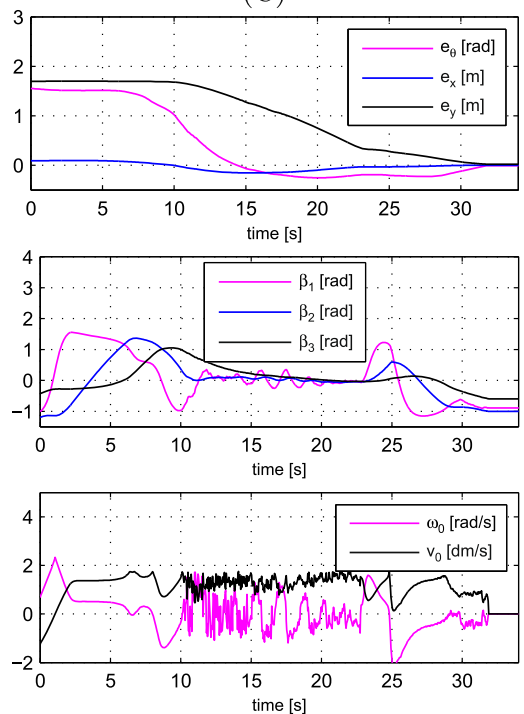

Fig. 9 E1: time-plots of particular signals for three forward parking maneuvers $(\mathbf{A}, \mathbf{B}$, and $\mathbf{C})$ performed with nS3T robot in the case of negative hitching offsets $\left(L_{h 1,2,3}<0\right)$ 


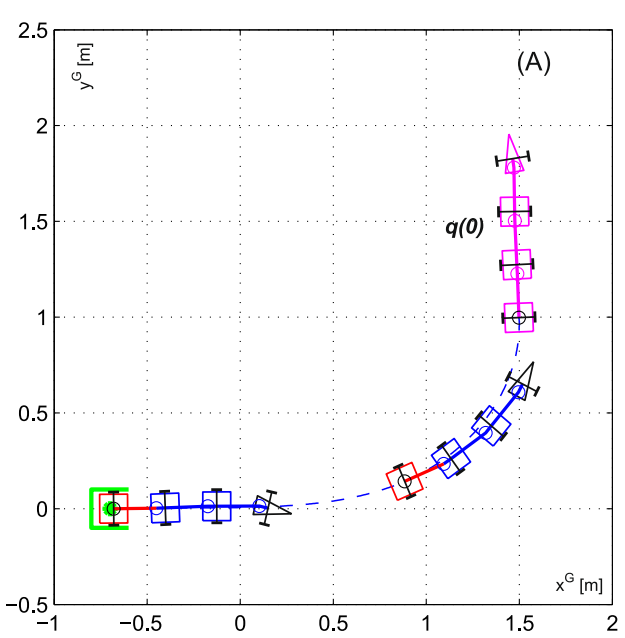

Fig. 10 E2: experimental results of three backward parking maneuvers ( $\mathbf{A}, \mathbf{B}$, and $\mathbf{C}$ ) performed in a task space with nS3T robot in the case of positive hitching offsets
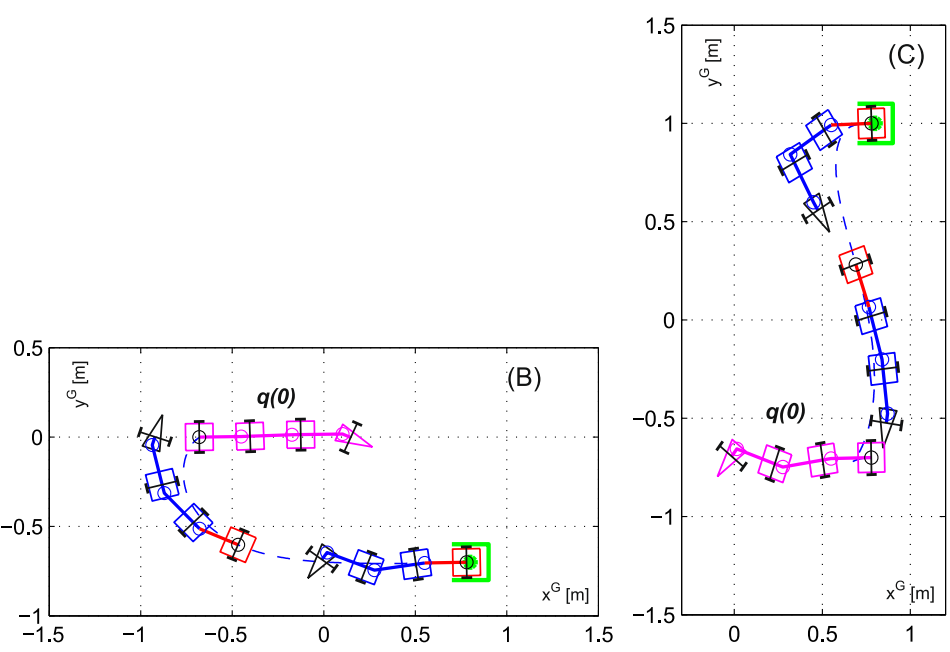

$\left(L_{h 1,2,3}>0\right)$; initial robot configurations $\boldsymbol{q}(0)$ are highlighted in magenta, and reference postures $\boldsymbol{q}_{3 r}$ are represented by green dock-marks

more, in spite of overestimated offsets used in the controller equations, the closed-loop system remained stable and control task has been successfully completed.

Experiment E2 For the purpose of experiment E2 the positive hitching offsets $L_{h 1}=L_{h 2}=$ $L_{h 3}=0.048 \mathrm{~m}$ were adjusted in the robot. In this case true offsets values were used in computations of the inner-loop controller and in the PbE. For the rest of controller parameters the following values were chosen: $k_{a}=2, k_{p}=1, \eta=0.8, \sigma=$ $-\operatorname{sgn}\left(L_{h i}\right)=-1$ (backward parking maneuvers), $\omega_{w \max }=6 \mathrm{rad} / \mathrm{s}$, and $w_{1}=0.98, w_{2}=0.02$. Similarly as in experiment E1 the switching strategy (Eq. 43) was used with $\delta=0.02$ and $w=\sqrt{0.001}$.
(A)
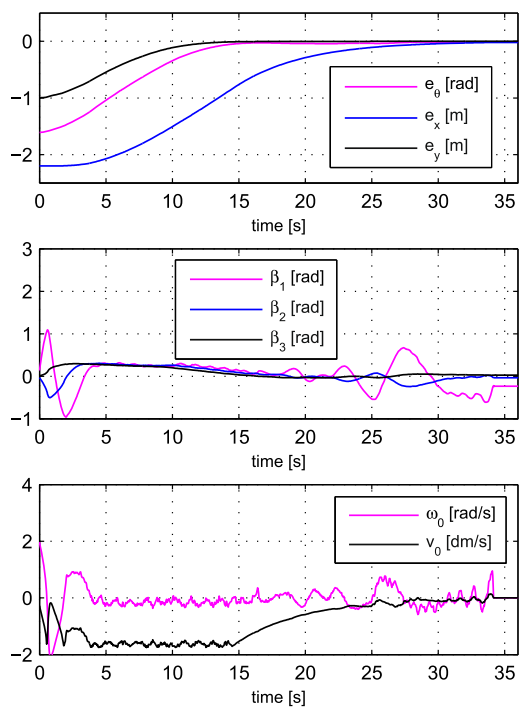

(B)
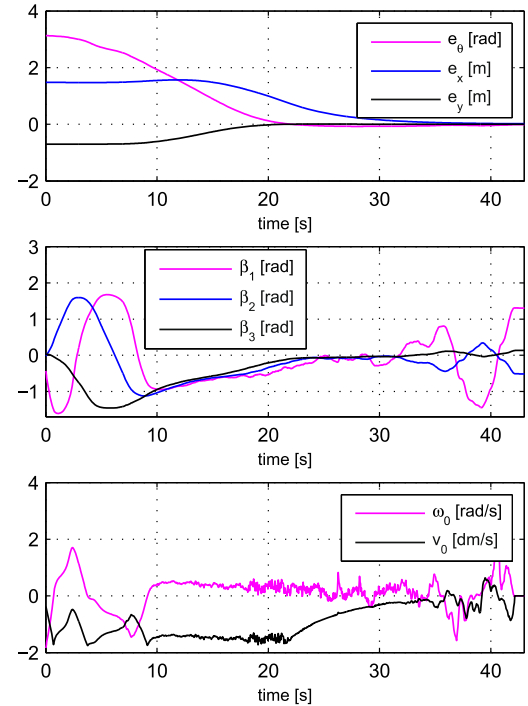

(C)
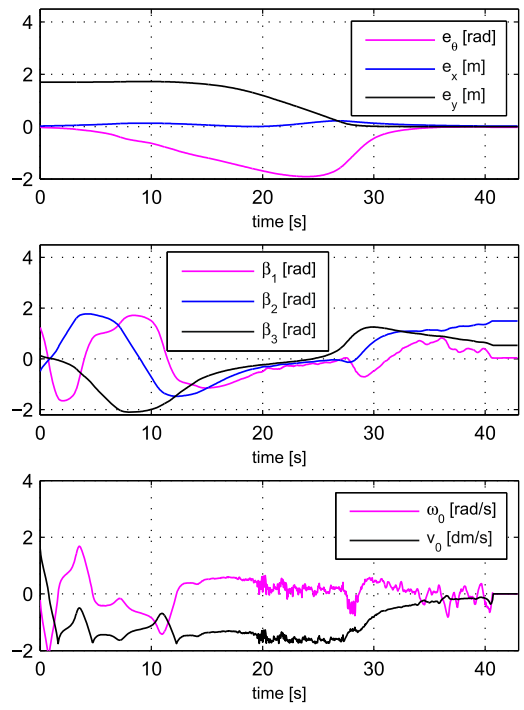

Fig. 11 E2: time-plots of particular signals for three backward parking maneuvers $(\mathbf{A}, \mathbf{B}$, and $\mathbf{C})$ performed with nS3T robot in the case of positive hitching offsets $\left(L_{h 1,2,3}>0\right)$ 
The results of experiment E2 are shown in Figs. 10 and 11. Three scenarios (A, B, and C) of subsequent parking maneuvers have been shown with the reference postures (values in $[\mathrm{rad}, \mathrm{m}, \mathrm{m}]$ )

$\boldsymbol{q}_{3 r}^{A}=\left[\begin{array}{c}0.0 \\ -0.7 \\ 0.0\end{array}\right], \quad \boldsymbol{q}_{3 r}^{B}=\left[\begin{array}{c}\pi \\ 0.8 \\ -0.7\end{array}\right], \quad \boldsymbol{q}_{3 r}^{C}=\left[\begin{array}{c}\pi \\ 0.8 \\ 1.0\end{array}\right]$

denoted in Fig. 10 by the green dock-marks.

Similarly as in experiment E1, non-oscillatory convergence of posture errors for the guidance segment can be seen in Fig. 11. This is a consequence of the VFO control action in the outer feedback loop. Control signals are bounded and preserve maximal admissible values $\omega_{0 \max }=$ $2.34 \mathrm{rad} / \mathrm{s}, v_{0 \max }=0.175 \mathrm{~m} / \mathrm{s}$. In contrast to results from experiment $\mathrm{E} 1$, in this case oscillations of control signals and joint angles are substantially reduced due to much longer hitching offsets adjusted in the RMP robot. Higher oscillations can be seen only in the terminal part of maneuvers as a result of sensitivity grow of VFO controller near point $\boldsymbol{e}=\mathbf{0}$ (see $[15,18]$ ). Worth to note the lack of any vehicle folding in spite of relatively high values of joint angles reached within transient states (especially for scenarios B and C).

\subsection{Results and Comments for Trajectory Tracking Task}

Control performance in the tracking task has been verified by two additional experiments-E3 and E4-conducted for elliptical and eight-shaped reference trajectories, respectively (both characterized by time-varying reference velocities). During experiments E3 and E4 the outer feedback loop was computing according to fusion mechanism (Eq. 42) with utilization of the PbE. Switching conditions, preventing indeterminacy in Eqs. 27 and 28 , was designed with small positive vicinity $\epsilon=0.02$ (cf. Remark 1).

Fig. 12 E3: experimental results of the forward trajectorytracking task (elliptical reference trajectory) with nS3T robot in the case of negative hitching offsets $\left(L_{h 1,2,3}<0\right)$; initial robot configuration $\boldsymbol{q}(0)$ is highlighted in magenta; the reference trajectory is denoted by the green dashed line
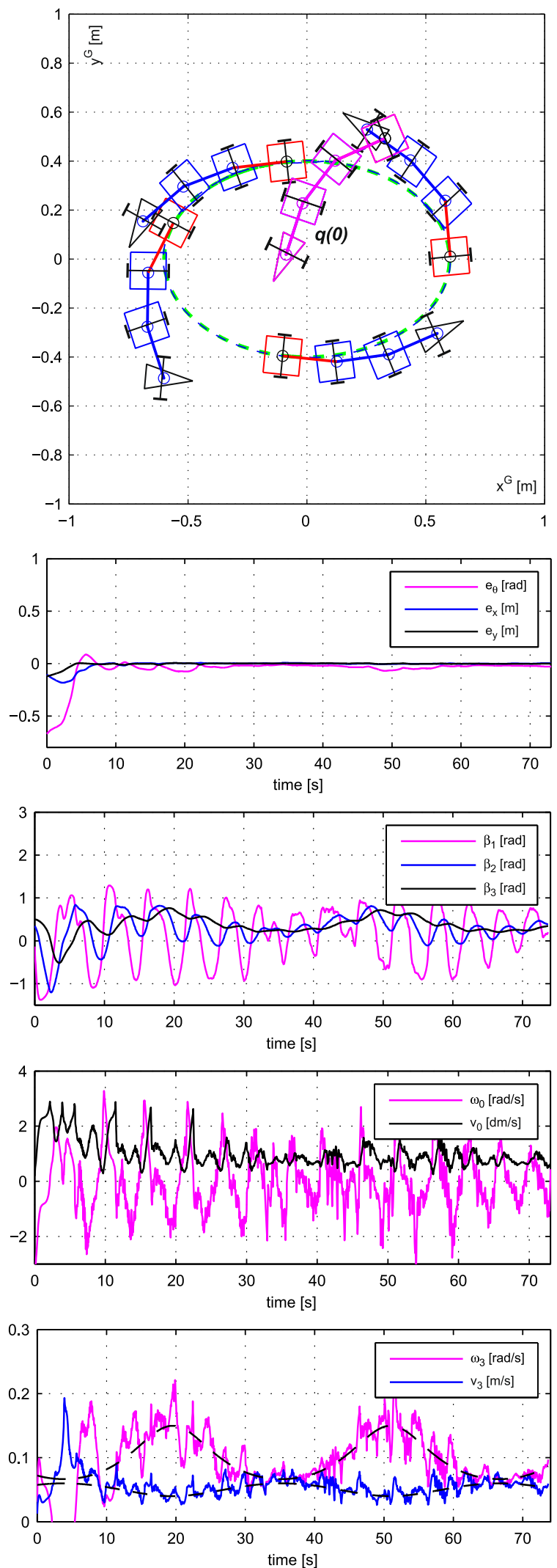
Fig. 13 E4: experimental results of the backward trajectory-tracking task (eight-shaped reference trajectory) with nS3T robot in the case of positive hitching offsets $\left(L_{h 1,2,3}>0\right)$; initial robot configuration $\boldsymbol{q}(0)$ is highlighted in magenta; the reference trajectory is denoted by the green dashed line

Experiment E3 In experiment E3, conducted with small negative hitching offsets $L_{h 1}=L_{h 2}=$ $L_{h 3}=-0.008 \mathrm{~m}$, the elliptical reference trajectory was selected, and determined by the timeparametrized equations (values in $[\mathrm{m}]): x_{3 r}(t)=$ $0.6 \cos (0.1 t), y_{3 r}(t)=0.4 \sin (0.1 t)$. Controller parameters and posture estimation weights were selected as follows: $k_{a}=2, k_{p}=1, \omega_{w \max }=10 \mathrm{rad} / \mathrm{s}$, $\sigma=-\operatorname{sgn}\left(L_{h i}\right)=+1$ (forward tracking) and $w_{1}=$ $0.95, w_{2}=0.05$. Similarly as for experiment E1 (and due to the same reasons), the overestimated values of hitching offsets $L_{h 1}=L_{h 2}=$ $L_{h 3}=-0.032 \mathrm{~m}$ were used in computations of the inner-loop controller and in the $\mathrm{PbE}$. The results of the elliptical trajectory tracking task are presented in Fig. 12. On the X-Y plot a reference path has been highlighted in green, path of the guidance segment has been denoted by blue dashed line, and initial robot configuration $\boldsymbol{q}(0)$ has been highlighted in magenta.

Due to the small hitching offsets adjusted in the robot the highly oscillatory tractor behavior can be observed according to the time-plots of joint angles and especially of control input $\omega_{0}(t)$. This effect, together with the fact that overestimated offsets values were used in computations, influenced the resultant tracking precision, especially for the orientation error $e_{\theta}$. Non-smooth motion of the last trailer can be also concluded based on the time plots of last-trailer velocities ${ }^{6}$ $\omega_{3}(t)$ and $v_{3}(t)$, which tracked the reference velocities (signals $\omega_{3 r}(t)$ and $v_{3 r}(t)$ denoted by black dashed lines in the bottom time-plot) only in the average sense. However, despite the oscillatory motion character, the rate of posture errors convergence and accuracy of position tracking (terminal behavior of errors $e_{x}(t)$ and $e_{y}(t)$ ) seem to be acceptable, and any vehicle folding is

\footnotetext{
${ }^{6}$ Velocities of the last trailer have been computed by using the tractor control inputs and propagation formula (11).
}
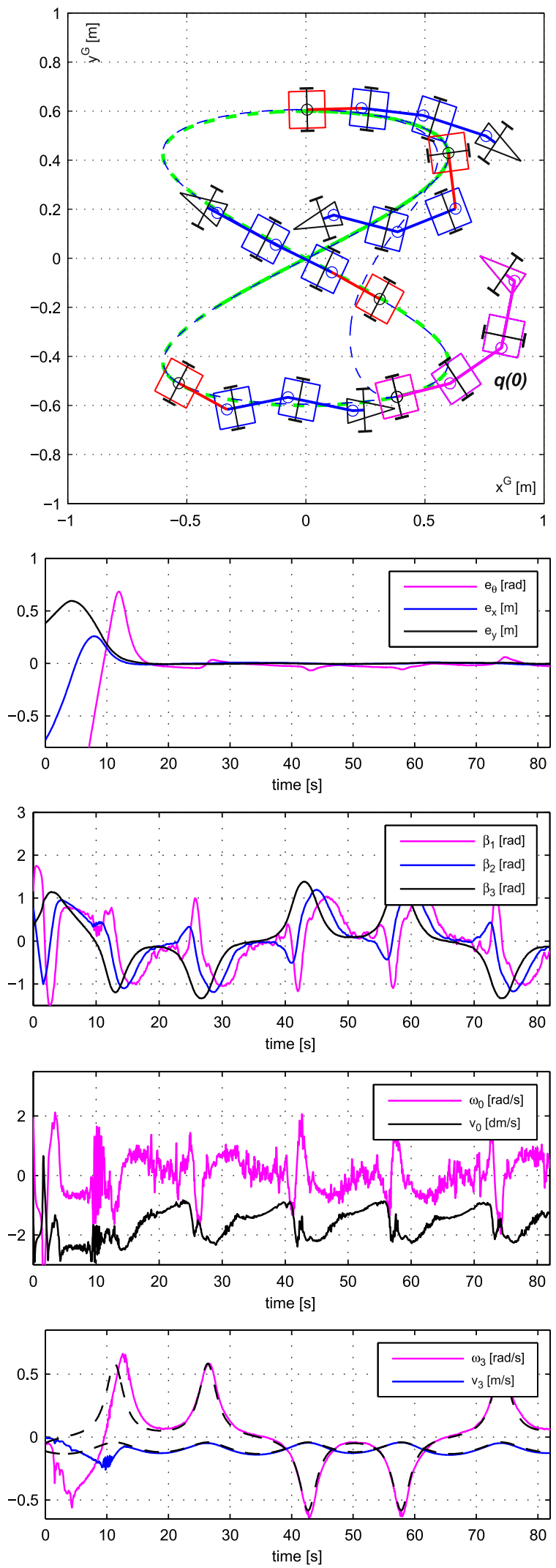
not present in the robot motion. During the whole control time-horizon the control signals are bounded and preserve maximal admissible values $\omega_{0 \max }=3.9 \mathrm{rad} / \mathrm{s}, v_{0 \max }=0.293 \mathrm{~m} / \mathrm{s}$.

Experiment E4 The last experiment was conducted using positive hitching offsets $L_{h 1}=L_{h 2}=$ $0.048 \mathrm{~m}$, and $L_{h 3}=0.032 \mathrm{~m}$. True offsets values were used in computations of the innerloop controller and in the $\mathrm{PbE}$. The reference eight-shaped trajectory was computing according to the time-parametrized equations (values in $[\mathrm{m}]): x_{3 r}(t)=0.6 \sin (0.2 t), y_{3 r}(t)=0.6 \sin (0.1 t)$. Controller parameters and posture estimation weights were selected as follows: $k_{a}=2, k_{p}=1$, $\omega_{w \max }=10 \mathrm{rad} / \mathrm{s}, \sigma=-\operatorname{sgn}\left(L_{h i}\right)=-1$ (backward tracking) and $w_{1}=0.98, w_{2}=0.02$. The results of the eight-shaped trajectory tracking task are presented in Fig. 13. On the X-Y plot a reference path has been highlighted in green, path of the guidance segment has been denoted by blue dashed line, and initial robot configuration $\boldsymbol{q}(0)$ has been highlighted in magenta.

Comparing the control performance obtained in this case with the results of experiment E3 one observes much less oscillations in the vehicle motion. This is a direct consequence of the longer hitching offsets adjusted in the robot. The resultant smooth behavior of the guidance segment can be seen on the plot of velocities ${ }^{7} \omega_{3}(t)$ and $v_{3}(t)$ which closely track the reference velocities $\omega_{3 r}(t)$ and $v_{3 r}(t)$ denoted by black dashed lines. Also in this case any folding effect did not occur during the tracking process. Bounded control signals preserved maximal admissible values $\omega_{0 \max }=$ $3.9 \mathrm{rad} / \mathrm{s}, v_{0 \max }=0.293 \mathrm{~m} / \mathrm{s}$.

\section{Conclusions}

The cascaded VFO control law, presented and practically validated in the paper, allows one to solve the trajectory tracking and set-point control tasks defined for a last trailer (guidance segment) of the non-standard $\mathrm{N}$-trailer robots. The concept

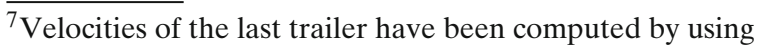
the tractor control inputs and propagation formula (11).
}

comes from treatment of N-trailer kinematics in the form of interconnected unicycle models with input-velocities resulting from tractor velocities propagated along a vehicle chain. Control performance obtained in the closed-loop system results from specific properties inherited from the VFO outer-loop controller. Experimental results presented in the paper have illustrated efficiency of the method in cases of backward and forward control strategies for positive and negative hitching offsets used in a vehicle.

According to numerous experiments conducted by the authors, and the results and comments provided in the paper (and in [15]) one can formulate some practical limitations of the proposed method:

- substantial grow of noise-sensitivity of the inner loop in the case of very small hitching offsets $L_{h i}$,

- a vehicle folding effect can be observed for the heterogeneous hitching of trailers (combined positive and negative hitching offsets in a vehicle); the folding effect can be avoided assuming the homogeneous hitching of trailers,

- motion control strategy (backward/forward) should be compatible with the signs of (homogeneous) hitching offsets to avoid folding of the vehicle.

Despite the above restrictions, several benefits of the concept seem to be justified-one can mention:

- unified treatment of trajectory tracking and set-point control tasks for backward as well as forward motion strategies,

- fast and non-oscillatory posture-error convergence for the guidance segment,

- a wide set of reference trajectories acceptable for the guidance segment (as a response of unicycle kinematics (Eq. 15) to persistently exciting reference inputs) without the need of reference signals generation for joint angles,

- simple addressing of tractor input limitations (by the velocity scaling procedure) and simplicity of the overall control scheme implementation,

- high scalability of the closed-loop system (adding additional trailers to a vehicle is not problematic from a control standpoint, since it 
only increases a number of matrices multiplied in Eq. 20).

Some important control issues still remain unsolved. The method proposed in the paper does not cope with mechanical constraints usually present in vehicle joints. In other words, we cannot guarantee that the joint angles of the $\mathrm{N}$-trailer stay in some prescribed limited ranges $\left(\beta_{i \min }, \beta_{i \max }\right)$, especially within a transient stage. The greatest challenge related to control of nSNT (and GNT) robots is to provide (practically acceptable) solution for a set-point stabilization of an arbitrary configuration $\boldsymbol{q}$ of the whole vehicle. In view of the very sparse literature on this topic (cf. for instance [13] and [35]), the problem seems to be still open.

Open Access This article is distributed under the terms of the Creative Commons Attribution License which permits any use, distribution, and reproduction in any medium, provided the original author(s) and the source are credited.

\section{References}

1. Altafini, C.: Some properties of the general n-trailer. Int. J. Control 74(4), 409-424 (2001)

2. Altafini, C.: Path following with reduced off-tracking for multibody wheeled vehicles. IEEE Trans. Control Syst. Technol. 11(4), 598-605 (2003)

3. Altafini, C., Speranzon, A., Wahlberg, B.: A feedback control scheme for reversing a truck and trailer vehicle. IEEE Trans. Robot. Autom. 17(6), 915-922 (2001)

4. Astolfi, A., Bolzern, P., Locatelli, A.: Path-tracking of a tractor-trailer vehicle along rectilinear and circular paths: a Lyapunov-based approach. IEEE Trans. Robot. Autom. 20(1), 154-160 (2004)

5. Bolzern, P., DeSantis, R.M., Locatelli, A., Masciocchi, D.: Path-tracking for articulated vehicles with off-axle hitching. IEEE Trans. Control Syst. Technol. 6(4), 515523 (1998)

6. Cariou, C., Lenain, R., Thuilot, B., Martinet, P.: Path following of a vehicle-trailer system in presence of sliding: application to automatic guidance of a towed agricultural implement. In: The 2010 IEEE/RSJ Int. Conf. Intel. Robots and Systems, pp. 4976-4981. Taipei, Taiwan (2010)

7. Chung, W., Park, M., Yoo, K., Roh, J.I., Choi, J.: Backward-motion control of a mobile robot with $n$ passive off-hooked trailers. J. Mech. Sci. Technol. 25(11), 2895-2905 (2011)

8. Cuesta, F., Gomez-Bravo, F., Ollero, A.: Parking maneuvers of industrial-like electrical vehicles with and without trailer. IEEE Trans. Ind. Electron. 51(2), 257269 (2004)
9. Ferrara, A., Magnani, L.: Hybrid variable structure path tracking control of articulated vehicles. In: Proc. of the 2004 American Control Conf., pp. 2777-2782. Boston, USA (2004)

10. Jean, F.: The car with $\mathrm{N}$ trailers: characterisation of the singular configurations. ESAIM: COCV 1, 241-266 (1996)

11. Lamiraux, F., Sekhavat, S., Laumond, J.: Motion planning and control for Hilare pulling a trailer. IEEE Trans. Robot. Autom. 15(4), 640-652 (1999)

12. Laumond, J.P.: Controllability of a multibody mobile robot. IEEE Trans. Robot. Autom. 9(6), 755-763 (1993)

13. Lizarraga, D.A., Morin, P., Samson, C.: Chained form approximation of a driftless system. Application to the exponential stabilization of the general n-trailer system. Int. J. Control 74(16), 1612-1629 (2001)

14. Matsushita, K., Murakami, T.: Nonholonomic equivalent disturbance based backward motion control of tractor-trailer with virtual steering. IEEE Trans. Ind. Electron. 55(1), 280-287 (2008)

15. Michalek, M.: Application of the VFO method to setpoint control for the $\mathrm{N}$-trailer vehicle with off-axle hitching. Int. J. Control 85(5), 502-521 (2012)

16. Michalek, M.: Non-minimum-phase property of Ntrailer kinematics resulting from off-axle interconnections. Int. J. Control 86(4), 740-758 (2013)

17. Michalek, M., Kozlowski, K.: Tracking and set-point VFO control for an articulated mobile robot with onaxle hitched trailer. In: 2009 American Control Conf., pp. 919-924. St. Louis, USA (2009)

18. Michalek, M., Kozlowski, K.: Vector-Field-Orientation feedback control method for a differentially driven vehicle. IEEE Trans. Control Syst. Technol. 18(1), 45-65 (2010)

19. Michalek, M., Kozlowski, K.: Convergence analysis for the orientation error of a unicycle in the VFO set-point control system. Authors' complementary note, pp. 1-5 (2012). Available on-line: http://etacar. put.poznan.pl/maciej.michalek

20. Morales, J., Martinez, J.L., Mandow, A., GarciaCerezo, A.J.: Steering the last trailer as a virtual tractor for reversing vehicles with passive on- and off-axle hitches. IEEE Trans. Ind. Electron. 60(12), 5729-5736 (2013)

21. Morales, J., Martinez, J.L., Mandow, A., Medina, I.J.: Virtual steering limitations for reversing an articulated vehicle with off-axle passive trailers. In: 35 th IEEE Annual Conf. of Industrial Electronics, pp. 2385-2390. Porto, Portugal (2009)

22. Morin, P., Samson, C.: Transverse function control of a class of non-invariant driftless systems. Application to vehicles with trailers. In: Proceedings of the 47th IEEE Conf. on Decision and Control, pp. 4312-4319. Cancun, Mexico (2008)

23. Niechwiadowicz, K., Kalaykov, I.: Behavior-based control of robot and trailer in hospitals. In: 18th Mediterranean Conf. on Control and Automation, pp. 1549-1554. Marrakech, Morocco (2010)

24. Orosco-Guerrero, R., Aranda-Bricaire, E., VelascoVilla, M.: Global path-tracking for a multi-steered gen- 
eral N-trailer. In: Proc. 15th IFAC Triennial World Congress. Barcelona, Spain (2002)

25. Park, M., Chung, W., Kim, M., Song, J.: Control of a mobile robot with passive multiple trailers. In: Proc. of the 2004 Int. Conf. Robotics and Automation, pp. 4369-4374. New Orleans, USA (2004)

26. Pazderski, D., Kozlowski, K., Waskowicz, D.: Control of a unicycle-like robot with trailers using transverse function approach. Bull. Pol. Ac.: Tech. 60(3), 537-546 (2012)

27. Pradalier, C., Usher, K.: Robust trajectory tracking for a reversing tractor trailer. Journal of Field Robotics 25(6-7), 378-399 (2008)

28. Ren, T.R., Kwok, N.M., Sui, C., Wang, D., Luo, J., $\mathrm{Su}, \mathrm{W} .:$ Controller design of a truck and multiple trailer system. In: Proc. of the 2010 IEEE Int. Conf. Robotics and Biomimetics, pp. 294-299. Tianjin, China (2010)

29. Rouchon, P., Fliess, M., Levine, J., Martin, P.: Flatness and motion planning: the car with $n$ trailers. In: Proc. European Control Conference, pp. 1518-1522. Groningen (1993)
30. Rouchon, P., Fliess, M., Levine, J., Martin, P.: Flatness, motion planning and trailer systems. In: Proceedings of the 32nd Conf. on Decision and Control, pp. 2700 2705. San Antonio, USA (1993)

31. Samson, C.: Control of chained systems. Application to path following and time-varying point-stabilization of mobile robots. IEEE Trans. Autom. Control 40(1), 64-77 (1995)

32. Sordalen, O.J., Wichlund, K.Y.: Exponential stabilization of a car with $n$ trailers. In: Proceedings of the 32th Conf. on Decision and Control, pp. 978-983. San Antonio, USA (1993)

33. Stahn, R., Heiserich, G., Stopp, A.: Laser scannerbased navigation for commercial vehicles. In: Proc. of the 2007 IEEE Intell. Vehicles Symposium, pp. 969974. Istanbul, Turkey (2007)

34. Tanaka, K., Hori, S., Wang, H.O.: Multiobjective control of a vehicle with triple trailers. IEEE/ASME T-Mech 7(3), 357-368 (2002)

35. Vendittelli, M., Oriolo, G.: Stabilization of the general two-trailer system. In: Proceedings of the 2000 IEEE Int. Conf. on Robotics and Automation, pp. 1817-1823. San Francisco, CA (2000) 